\title{
AVALIAÇÃO DO POTENCIAL ANTICORROSIVO DE TIOSSEMICARBAZONAS SOLUBILIZADAS EM MICROEMULSÃO
}

Elaine Cristina M. de Moura, Adna Dornelles N. Souza, Cátia Guaraciara F. T. Rossi, Djalma R. da Silva e Maria Aparecida M. Maciel*

Instituto de Química, Universidade Federal do Rio Grande do Norte, Campus Universitário, 59078-970 Natal - RN, Brasil Aurea Echevarria e Myrtes S. S. Bellieny

Departamento de Química, Instituto de Ciências Exatas, Universidade Federal Rural do Rio de Janeiro, 23851-970 Seropédica RJ, Brasil

Recebido em 8/3/12; aceito em 27/7/12; publicado na web em 26/11/12

\begin{abstract}
EVALUATION OF ANTICORROSIVE EFFECTIVENESS OF THIOSEMICARBAZONES SOLUBILIZED IN A MICROEMULSION SYSTEM. In this paper, thiosemicarbazones 4-N-cinnamoyl-thiosemicarbazone (CTSC), 4-N-(2'methoxycinnamoyl)-thiosemicarbazone (MCTSC), and 4-N-(4'-hydroxy-3'-methoxybenzoyl)- thiosemicarbazone (HMBTSC) were solubilized in an oil-in-water (O/W) microemulsion system (ME_OCS), forming systems CTSC_ME_OCS, MCTSC_ME_OCS and HMBTSC_ME_OCS. The effectiveness of these systems in the process of inhibiting AISI 1020 carbon steel corrosion was evaluated in a saline solution $(\mathrm{NaCl} 0.5 \%)$, using a galvanostatic method. The tested thiosemicarbazones showed higher inhibitory effects (85.7\% for CTSC_ME_OCS, 84.0\% for MCTSC_ME_OCS, and 83.3\% for HMBTSC_ME_OCS). The surfactant OCS (dissolved in $\mathrm{H}_{2} \mathrm{O}$ ) and the ME_OCS system showed lower efficacies, with $71.0 \%$ for OCS and $74.0 \%$ for ME_OCS system.
\end{abstract}

Keywords: thiosemicarbazones; microemulsion; corrosion inhibitors.

\section{INTRODUÇÃo}

Os problemas causados por processos corrosivos são de ocorrência frequente nas mais variadas atividades desenvolvidas nas indústrias químicas, petrolíferas, petroquímicas, naval, construção civil, dentre outras. ${ }^{1}$

A utilização de tensoativos aplicados em processos que avaliam a redução de corrosão sofre sistematicamente avanços tecnológicos, como pode ser evidenciado em diversos trabalhos..$^{2-10}$ Com relação ao uso do tensoativo óleo de coco saponificado, Rossi et $a l .{ }^{4}$ avaliaram a eficiência de inibição à corrosão em aço AISI 1020 por métodos eletroquímicos (curvas de polarização e galvanostático). Este tensoativo foi aplicado em um sistema microemulsionado com resultados significativos.

Inibidores químicos de caráter inorgânico e/ou orgânico são amplamente utilizados por agirem formando películas protetoras sobre áreas anódicas e catódicas, controlando desta forma reações eletroquímicas. ${ }^{11}$ Especificamente, alguns derivados de tiossemicarbazonas vêm sendo testados como potentes inibidores de corrosão. ${ }^{12-16}$

Neste trabalho, os compostos 4-N-cinamoil-tiossemicarbazona (CTSC), 4-N(2'-metoxicinamoil)-tiossemicarbazona (MCTSC) e 4-N-(4'-hidroxi-3'-metoxibenzoil)-tiossemicarbazona (HMBTSC) foram solubilizados no sistema microemulsionado ME_OCS para aplicabilidade como inibidores de corrosão. As eficácias das microemulsões contendo as tiossemicarbazonas CTSC_ME_OCS, MCTSC_ME_OCS e HMBTSC_ME_OCS foram avaliadas em aço carbono AISI 1020, em meio salino, pelo método galvanostático.

\section{PARTE EXPERIMENTAL}

As análises físico-química do óleo de coco, a obtenção do óleo de coco saponificado (OCS) e do sistema microemulsionado ME_OCS foram realizados de acordo com metodologia previamente

*e-mail: mammaciel@hotmail.com reportada. ${ }^{4,17}$ As tiossemicarbazonas avaliadas foram previamente sintetizadas, caracterizadas e reportadas para aplicabilidade biológica. ${ }^{18,19}$

\section{Solubilização de tiossemicarbazonas em microemulsão}

A eficácia do sistema microemulsionado na solubilização das tiossemicarbazonas CTSC, MCTSC e HMBTSC foi avaliada pelo método espectroscópico no ultravioleta, utilizando um comprimento de onda ( $\lambda$ ) na faixa 200 a $400 \mathrm{~nm}$, para determinação do $\lambda_{\text {máx }}$. Para obtenção da curva analítica, que consiste na relação entre a concentração e a absorbância de 5 amostras em um determinado $\lambda_{\text {máx }}$, utilizou-se uma massa conhecida de cada uma das tiossemicarbazonas (Tabela 1) solubilizadas em 1,0 mL do sistema ME_OCS. As amostras foram preparadas pela adição das tiossemicarbazona no ME_OCS até a ocorrência de corpo de fundo. Após rigorosa agitação (intercalando intervalos de repouso) foram obtidas leituras (em triplicata) de acordo com o $\lambda_{\text {máx }}$ determinado anteriormente e, quando necessário, foram realizadas diluições com ME_OCS. Desta forma, o valor de absorbância da amostra em análise é lida no espectrofotômetro e a sua concentração é obtida via curva analítica, através do ponto de intersecção.

\section{Medidas de eficiência de inibição à corrosão}

O estudo das eficiências de inibição à corrosão de tiossemicarbazonas solubilizadas em microemulsão em meio salino foi realizado em célula clássica de três eletrodos: o de referência em $\mathrm{Ag} / \mathrm{AgCl}$ (prata/cloreto de prata), o auxiliar de platina e o de trabalho, em aço carbono AISI 1020. Nesta célula, foi inserido um borbulhador de $\mathrm{O}_{2}$ objetivando promover oxigenação contínua no sistema, tendo sido utilizado o método eletroquímico galvanostático para as medições das eficiências de inibições à corrosão. Neste método, a corrente controlada pelo galvanostato é aplicada ao eletrodo de trabalho e possibilita a medição da diferença de potencial elétrico entre os eletrodos de trabalho e de referência. O sistema eletroquímico galvanostático é 
Tabela 1. Nomenclatura e estrutura química das tiossemicarbazonas avaliadas

4-N-cinamoil-tiossemicarbazona

constituído por um resistor em série com um capacitor que recebe corrente elétrica que varia linearmente com o tempo, $i(t)$, de acordo com a Equação 1:

$$
i(t)=i_{0}+\lambda t
$$

em que $i_{0}$ corresponde à corrente inicial e $\lambda$ à taxa de variação da corrente com o tempo. Desta forma, a diferença de potencial, $V$, pode ser estimada considerando-se que este parâmetro é uma função quadrática do tempo e cujos coeficientes lineares estão relacionados com os parâmetros do sistema eletroquímico utilizado, de acordo com a Equação 2:

$$
V(i)=-\frac{i_{0}^{2}}{2 \lambda \mathrm{C}}+R \cdot i+\frac{i^{2}}{2 \lambda \mathrm{C}}
$$

em que $R$ e $\mathrm{C}$ correspondem à resistência e capacitância do sistema eletroquímico, respectivamente. Para cada concentração de inibidor calcula-se a resistência elétrica $R$, bem como a eficiência de inibição $(\theta)$. As modificações de eficiência de inibição estão correlacionadas com as concentrações do inibidor avaliado e são função da resistência elétrica $R$ do sistema eletroquímico, que pode ser dada pela Equação 3:

$$
\theta=\frac{\left(R-R_{0}\right)}{R}
$$

em que $\theta$ corresponde à eficiência de inibição, $R$ ao valor da resistência medida na presença do inibidor e $R_{0}$ é o valor da resistência medida na ausência do inibidor.

De modo a se avaliar o processo de adsorção, as isotermas de adsorção de Langmuir e Frumkin são dadas pelas Equações 4 e 5, respectivamente:

$$
\begin{gathered}
\frac{\theta}{(1-\theta)}=K C \\
\frac{\theta}{(1-\theta)}=K C \exp (A \theta)
\end{gathered}
$$

em que $K$ corresponde à constante de adsorção, $A$ é o parâmetro de interação lateral e $C$ é a concentração do inibidor.

Os parâmetros $K$ (constante de adsorção) e $A$ (interação lateral) foram determinados utilizando-se a minimização direta a partir da Equação 6 (com um software em Q-Basic ${ }^{\circledR}$ do método de otimização simplex Nelder-Mead). ${ }^{20}$ Este método consiste em determinar os valores dos parâmetros das isotermas a partir da eficiência de inibição observada $\left(\theta_{o b s}\right)$ versus eficiência de inibição calculada $\left(\theta_{\text {cal }}\right)$ (Equação 6).

$$
F(K, A)=\sum_{i=1}^{\mathrm{N}}\left(\frac{\theta_{\mathrm{cal}, \mathrm{i}}-\theta_{\mathrm{obs}, \mathrm{i}}}{\theta_{\mathrm{obs}, \mathrm{i}}}\right)^{2}
$$

A eficiência de corrosão $(E)$ foi determinada pela Equação 7.

$$
E(\%)=\frac{\left(R-R_{0}\right)}{R} \times 100
$$

\section{RESULTADOS E DISCUSSÃO}

\section{Solubilização de tiossemicarbazonas em microemulsão}

De forma geral, tiossemicarbazonas apresentam pouca solubilidade em meio aquoso, porém a disponibilidade desta classe de substância orgânica pode ser otimizada com a utilização de sistemas microemulsionados que apresentam elevada estabilidade e poder solubilizante. ${ }^{4,17}$

A quantificação da eficácia de solubilização do sistema microemulsionado ME_OCS para as tiossemicarbazonas avaliadas foi determinada após sucessivas diluições nesta microemulsão, por espectroscopia na região do ultravioleta (UV), obtendo-se curva de calibração para cada substância (Tabela 2). A estabilização da microemulsão foi confirmada pelos valores de tensão superficial, tendo sido observado que em diluições sucessivas não ocorreu quebra da região de microemulsão, caracterizando este sistema como autoemulsificante. Resultado semelhante foi conseguido diluindo-se o sistema ME_OCS em meio aquoso salino ( $\mathrm{NaCl}$ 0,5\%). Em função da eficácia de solubilização das tiossemicarbazonas (29,0-91,0\% de eficácia de solubilização) e da estabilidade da microemulsão em meios aquoso e salino, avaliou-se a eficiência de inibição à corrosão de CTSC, MCTSC e HMBTSC incorporadas, separadamente, neste sistema autoemulsificante, resultando nas seguintes formulações: CTSC_ME_OCS, MCTSC_ME_OCS e HMBTSC_ME_OCS que foram avaliadas pelo método galvanostático.

\section{Medidas de eficiência de inibição à corrosão}

O sistema ME_OCS foi obtido com o tensoativo óleo de coco saponificado (20,0\%), cotensoativo butan-1-ol (20,0\%), fase aquosa $(55,0 \%)$ e fase oleosa ( $5,0 \%$ de querosene), de acordo com metodologia previamente reportada. ${ }^{4}$ Os sistemas microemulsionados contendo as tiossemicarbazonas (Tabela 2) foram preparados de acordo com as máximas de solubilidade de cada substância, tendo sido escolhido um ponto na região de WIV polar (região rica em água). Para a realização dos ensaios eletroquímicos preparou-se uma solução suporte de $\mathrm{NaCl}$ padrão a $0,5 \%$ em massa. As massas de cada tiossemicarbazona avaliada nas análises de eficiência de inibição à corrosão encontram-se descritas na Tabela 3. 
Tabela 2. Dados de solubilização das tiossemicarbazonas avaliadas na microemulsão

\begin{tabular}{cccc}
\hline $\begin{array}{c}\text { Tiossemicarba- } \\
\text { zona }\end{array}$ & $\lambda_{\text {máx }}$ & $\begin{array}{c}\text { Solubilidade em } \\
\text { mol L }\end{array}$ & $\begin{array}{c}\% \text { de } \\
\text { solubilidade }\end{array}$ \\
\hline CTSC & 335 & $9,47 \times 10^{-3} \pm 7,54 \times 10^{-4}$ & 32,0 \\
MCTSC & 345 & $2,82 \times 10^{-3} \pm 6,18 \times 10^{-5}$ & 28,9 \\
HMBTSC & 355 & $1,17 \times 10^{-2} \pm 2,44 \times 10^{-5}$ & 91,0 \\
\hline
\end{tabular}

* Os valores correspondem à média de 3 experimentos

Tabela 3. Massas de tiossemicarbazonas utilizadas para teste de inibição à corrosão

\begin{tabular}{ccc}
\hline Tiossemicarbazona & Massa $(\mathrm{g})$ & $\begin{array}{c}\text { Volume de ME_OCS } \\
(\mathrm{mL})\end{array}$ \\
\hline CTSC & 0,214 & 110,0 \\
MCTSC & 0,073 & 110,0 \\
HMBTSC & 0,290 & 110,0 \\
\hline
\end{tabular}

As eficiências máximas de inibições observadas foram analisadas por comparação utilizando-se as isotermas de Langmuir e Frumkin (Figura 1a: 85,7\% para CTSC_ME_OCS, 1b: 84,0\% para MCTSC_ ME_OCS e 1c: 83,3\% para HMBTSC_ME_OCS).

Comparativamente, observou-se que a isoterma de Frumkin (com parâmetros discriminados na Tabela 4) aproxima-se mais dos dados experimentais do que a isoterma de Langmuir (Figura 1). $\mathrm{Na}$ isoterma de Frumkin, as constantes de adsorção $(K)$ e os parâmetros de interação lateral $(A)$ de CTSC_ME_OCS, MCTSC_ME_OCS e HMBTSC_ME_OCS apresentaram melhores resultados.

Tabela 4. Constante de adsorção $(K)$, parâmetros de interação lateral $(A)$ e eficiência de inibição $(E)$

\begin{tabular}{lccccc}
\hline & \multicolumn{2}{c}{ LANGMUIR } & \multicolumn{2}{c}{ FRUMKIN } & \multirow{2}{*}{$E(\%)$} \\
\cline { 2 - 5 } & $K$ & $A$ & $K$ & $A$ & \\
\hline OCS & $4,42 \times 10^{+2}$ & ---- & $4,31 \times 10^{+2}$ & $-0,06$ & 71,0 \\
ME_OCS & $3,85 \times 10^{+2}$ & ---- & $2,36 \times 10^{+2}$ & $-1,55$ & 74,0 \\
CTSC_ME_OCS & $0,95 \times 10^{+1}$ & ---- & $1,31 \times 10^{+3}$ & 6,91 & 85,7 \\
MCTSC_ME_OCS & $1,21 \times 10^{+1}$ & ---- & $1,23 \times 10^{+5}$ & 12,2 & 84,0 \\
HMBTSC_ME_OCS & $0,69 \times 10^{+1}$ & ---- & $2,84 \times 10^{+1}$ & 2,26 & 83,3 \\
\hline
\end{tabular}

Os valores negativos obtidos para o parâmetro $A$ (interação lateral entre as moléculas) do tensoativo OCS e do sistema autoemulsificante ME_OCS, bem como para as microemulsões contendo as tiossemicarbazonas, sugerem a ocorrência de interações laterais entre as moléculas testadas. Este tipo de interação pode ser justificado pelas forças de repulsão entre a parte polar do tensoativo e as cargas geradas nos híbridos de ressonância de cada substância.

Os resultados obtidos para os sistemas CTSC_ME_OCS, MCTSC_ME_OCS e HMBTSC_ME_OCS foram comparados com dados obtidos preliminarmente ${ }^{4}$ para as eficiências do tensoativo OCS e do sistema ME_OCS, através do método galvanostático (Tabela 4). As diferenças observadas nas constantes de adsorção $(K)$ de CTSC_ME_OCS $\left(1,31 \times 10^{+3}\right)$, MCTSC_ME_OCS $\left(1,23 \times 10^{+5}\right)$ e HMBTSC_ME_OCS $\left(2,84 \times 10^{+1}\right)$ podem ser justificadas pelas diferenças estruturais destas tiossemicarbazonas.

$\mathrm{O}$ fato de as tiossemicarbazonas não terem sido solúveis em meio neutro ou salino impossibilitou a realização de testes comparativos, dificultando esclarecimentos mais precisos sobre a eficiência real de cada substância (CTSC, MCTSC e HMBTSC) não veiculada. No
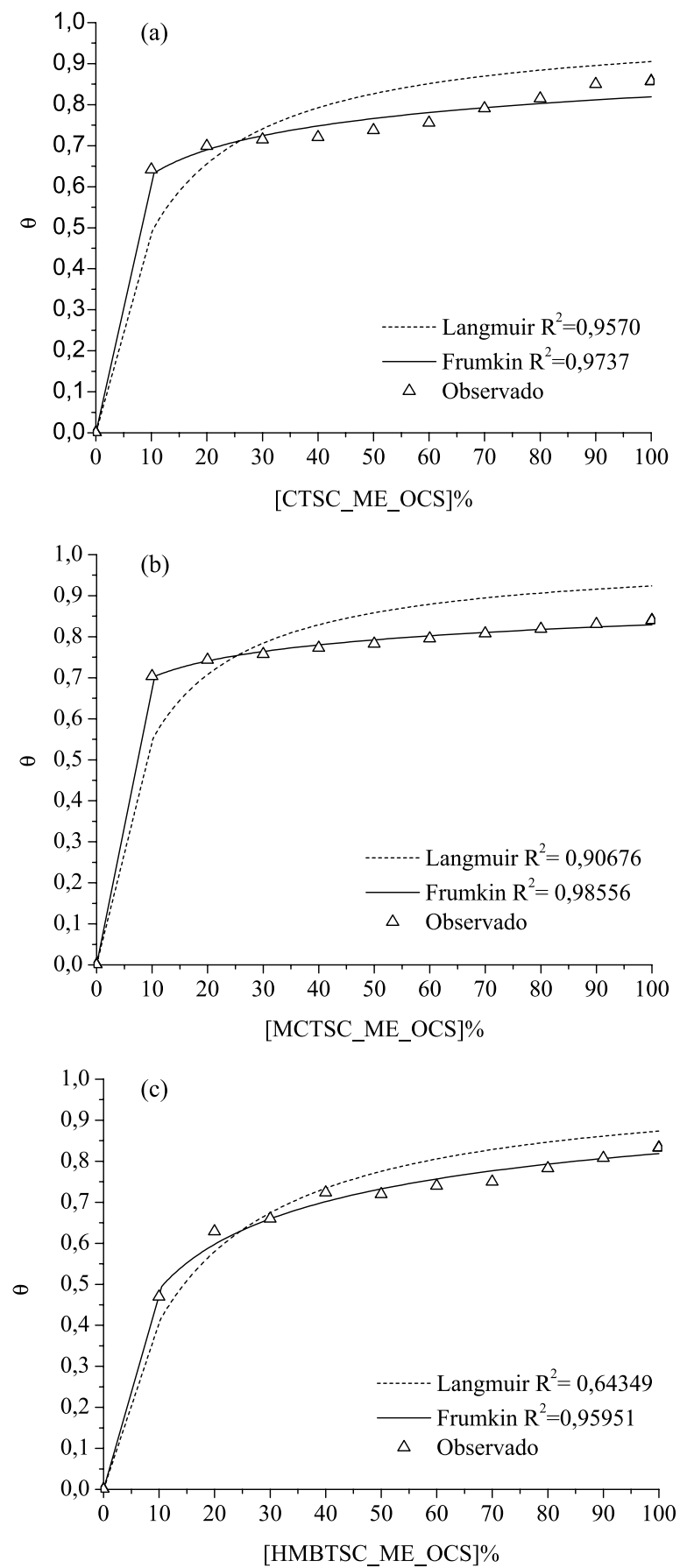

Figura 1. Isotermas de Frumkin e Langmuir: (a) CTSC_ME_OCS; (b) $M C T S C \_M E \_O C S$; (c) HMBTSC_ME_OCS

entanto, estudos anteriores mostraram que a CTSC (4-N-cinamoiltiossemicarbazona) solubilizada em meio corrosivo mais agressivo (meio ácido) apresentou eficácia máxima de inibição à corrosão em torno de $99,0 \%\left(10^{-4} \mathrm{M}\right){ }^{21-23}$ Observou-se também que substâncias orgânicas ricas em heteroátomos (oxigênio e nitrogênio) e em anel aromático são inibidoras eficientes por apresentarm elevada distribuição de cargas, facilitando a adsorção. ${ }^{12}$

\section{CONCLUSÕES}

As constantes de adsorção de Frumkin (K) obtidas para OCS (solubilizado em água e em meio salino) e ME_OCS, bem como para as tiossemicarbazonas solubilizadas nesta microemulsão, sofrem 
variações em função do meio aquoso [água destilada e solução salina $(\mathrm{NaCl})]$; as substâncias testadas em baixas concentrações $(0,214 \mathrm{~g}$ para CTSC; 0,073 g para MCTSC e 0,290 g para HMBTSC) veiculadas na microemulsão polar autoemulsificante ME_OCS apresentaram inibições significativas à corrosão (faixa de variação 85,7-83,3\%).

A comparação entre os parâmetros de interação lateral $(A)$ das tiossemicarbazonas (Tabela 4) com o tensoativo OCS e com o sistema ME_OCS sugere que a ocorrência de interação lateral (atrativa) é significativamente maior para as tiossemicarbazonas em função da maior adsorção entre as tiossemicarbazonas e a superfície metálica com adsorção do tipo líquido-sólido (sistema microemulsionado-metal).

A presença de heteroátomos e das subunidades aromáticas gera uma série significativa de híbridos de ressonância (espécies carregadas) que promovem uma maior interação da molécula com a superfície metálica, favorecendo o fenômeno de adsorção com melhores resultados de inibição à corrosão. Desta forma, justifica-se a maior eficiência de inibição à corrosão dos sistemas microemulsionados testados [CTSC-ME-OCS (85,7\%), MCTSC_ME_OCS $(84,0 \%)$ e HMBTSC_ME_OCS $(83,3 \%)]$ por comparação com o sistema OCSME $(74,0 \%)^{4}$ isento de tiossemicarbazona.

Neste trabalho, confirmou-se que CTSC, MCTSC e HMBTSC solubilizadas na microemulsão ME_OCS e avaliadas em baixas concentrações potencializam a eficiência de inibição em meio salino. As características estruturais de cada substância veiculada nesta microemulsão não interferem significantemente nas máximas de inibição. Em adição, fica confirmado que sistemas microemulsionados contribuem com os estudos que avaliam a disponibilização de substâncias orgânicas de difícil solubilização para posteriores aplicabilidades, a eficiência de inibidores de corrosão orgânicos e a utilização de microemulsões, bem como incentivam novas propostas de pesquisas para o aproveitamento da matéria orgânica vegetal óleo de coco, que atualmente é alvo de vários estudos em biotecnologia.

\section{REFERÊNCIAS}

1. Gentil, A.; Corrosão, $6^{\mathrm{a}}$ ed., LTC Livros Técnicos e Científicos S.A.: Rio de Janeiro, 2011.

2. Dantas, T. N. C.; Moura, E. F.; Scatena Júnior, H.; Dantas Neto, A. A.; Corrosion 2002, 58, 723 .
3. Wanderley Neto, A. O.; Moura, E. F.; Scatena Júnior, H.; Dantas, T. N. C.; Dantas Neto, A. A.; Gurgel, A.; Colloids Surf., A 2012, 398, 76.

4. Rossi, C. G. F. T.; Scatena Júnior, H.; Maciel, M. A. M.; Dantas, T. N. C.; Quim. Nova 2007, 30, 1128.

5. Rossi, C. G. F. T.; Dantas, T. N. C.; Dantas Neto, A. A.; Maciel, M. A. M.; Rev. Univ. Rural, Sér. Ciênc. Exatas Terra 2007, 26, 45.

6. Shiao, S. V.; Chhabra, V.; Patist, A.; Free, M. L.; Huibers, P. D. T.; Adv. Colloid Interface Sci. 1998, 74, 1.

7. Brolo, A. G.; Temperini, M. L. A.; Agostinho, S. M. L.; Electrochim. Acta 1998, 44, 559.

8. Luo, H.; Guan, Y. C.; Han, K. N.; Corrosion 1998, 54, 721.

9. El-Achouri, M.; Hajji, M. S.; Salem, M.; Kertit, S.; Aride, J.; Coudert, R.; Essassi, E. M.; Corros. Sci. 1996, 52, 103.

10. Mu, G. N.; Zhao, T. P.; Liu, M.; Gu, T.; Corrosion 1996, 52, 853.

11. Moura, E. F.; Wanderley Neto, A. O.; Dantas, T. N. C.; Scatena Júnior, H.; Gurgel, A.; Colloids Surf., A 2009, 340, 199.

12. Ebenso, E. E.; Ekpe, U. J.; Ita, B. I.; Offing, O. E.; Ibok, U. J.; Mater. Chem. Phys. 1999, 60, 79.

13. Ekpe, U. J.; Ibok, U. J.; Ita, B. I.; Offing, O. E.; Ebenso, E. E.; Mater. Chem. Phys. 1995, 40, 87

14. Lukovits, I.; Shaban, A.; Kálmán, E.; Electrochim. Acta 2005, 50, 4128.

15. Barakat, Y. F.; Hassan, A. M.; Baraka, A. M.; Materialwis. Werkst. 2000 , 31, 175.

16. Khamis, E.; Ameer, M. A.; Al-Andios, N. M.; Al-Senani, G.; Corrosion 2000, 52, 127.

17. Dantas, T. N. C.; Dantas Neto, A. A.; Moura, M. C. P. A.; Water Res. 2001, 35, 2219

18. Soares, R. O. A.; Echevarria, A.; Bellieny, M. S. S.; Pinho, R. T.; Leo, R. M. M.; Seguins, W. S.; Machado, G. M.; Canto-Cavalheiro, M. M.; Leon, L. L.; Exp. Parasitol. 2011, 129, 381.

19. Reis, C. M.; Pereira, D. S.; Paiva, R. O.; Kneipp, L. F.; Echevarria, A.; Molecules 2011, 16, 10668.

20. Prosser, A. J.; Franses, E. I. ; Colloids Surf., A 2001, 178, 1.

21. Ameer, M. A.; Khamis, E.; Al-Senani, G.; Prevention of metal corrosion: new research, Nova Science Publishers, lnc.: Portland, 2007.

22. Quraishi, M. A.; Jamal, D.; Mater. Performace 2002, 41, 38.

23. Jamal, D.; Quraishi, M. A.; J. Electrochem. Soc. India 2000, 49, 56. 